\title{
Zur Frage der postoperativen Azotämie und Azoturie, mit besonderer Berücksichtigung ihrer diuretischen Beeinflussung bei Bauchoperationen.
}

\author{
Von \\ Masatosi Shiozawa. \\ (留 濯 正俊) \\ Direktor: Prof. D. M. Muto.)
}

(Aus der chirurgischen Klinik der Universität, Sendai.

(Received for publication, November 29, 1944)

Ich beschäftigte mich mit einigen Stickstoffbestimmungen des Blutes und des Harns bei einer verhältnismässig grossen Anzahl von Bauchoperationen vor und nach dem Eingriff. Hier will ich kurz berichten über einige bemerkenswerte Eergbnisse, die ich aus meinen Untersuchungen an 79 Operierten, bei denen der Eingriff ohne postoperative Komplikationen glatt verheilte, gewonnen habe.

\section{Untersuchungsmethode.}

Ich bestimmte bei Operierten in unserer Klinik den Rest- und Urea$\mathrm{N}$ des Blutes sowie den Gesamt- und Urea-N des Harns 1 mal innerhalb einiger Tage vor und 4 mal, d.h. 1 u. 3. Tage, sowie 1 u. 2 Wochen nach der Operation. Der Rest-N des Blutes und der Gesamt-N des Harns wurden nach der Mikrokjeldahlmethode von Pregel, der Urea-N des Blutes und des Harns nach der Ureasemethode von Cullen-V an Slyke bestimmt. Zur Bestimmung dieser N-Substanzen im Blut wurde mit Natriumcitrat behandeltes Vollblut benutzt. Zur Enteiweissung des Blutes und des eiweisshaltigen Harns gebrauchte ich 10\%ige Trichloressigsäure.

Bei der Hälfte der Fälle spritzte ich während des Untersuchungsverlaufes in jeder Operationsgruppe täglich ausser einer grossen Menge Flüssigkeit 1 oder $2 \mathrm{~g}$ spezifisch harntreibende Mittel von Purin- und Quecksilberpräparaten, wie Coffeinum natriobenzoicum, Novasurol und Igrosin ein, um zu sehen, ob etwa bemerkenswerte Unterschiede der N-Werte des Blutes und des Harns zwischen den so behandelten Fällen und den anderen ohne Injektion von specifisch diuretischen Mitteln bestünden. Stra ten's Vorschlag") hat mich dazu veranlasst. Bei meinen 
Untersuchungen konnt ich, wenn ich auch die von ihm empfohlenen Präparate nicht benutzen konnte, an den N-Spiegeln des Blutes und des Harns keinerlei ausgeprägte Unterschiede zwischen den beiden Versuchsreihen feststellen. Es liegt sehr nahe, dass die grösseren Mengen Flüssigkeit bei meinen Fällen schon ohne spezifische Diuretica so stark harntreibend wirkten, dass die von mir gebrauchten Präparaten ihre Wirkung nicht zureichend ausüben konnten. Deshalb werde ich bei Darstellung meiner Ergebnisse die Angabe der spezifischen Diuretica der Kürze halber unterlassen. Bei meinen Fällen war der positive Einfluss der Nahrung auf die Vermehrung der N-haltigen Substanzen des Blutes und des Harns sicher ausgeschlossen, so dass ich bei der folgenden Darstellung auch keine Angaben über die Nahrung bringe.

\section{Ergebnisse meiner Untersuchungen.}

1. Untersuchungen an gesunden Nichtoperierten.

Über die Werte des Rest- und des Urea-N im Blute von Gesunden ist von $\mathrm{Kaya}^{2)}$ in unserer Klinik ausführlich berichtet worden. Bei meinen Untersuchungen am 18 Gesunden betrug der Wert des Rest-N durchschnittlich $31 \mathrm{mg}$ mit den Grenzwerten von $24 \mathrm{mg}$ und $35 \mathrm{mg}$ in $100 \mathrm{ccm}$ Blut, der Urea-N durchschnittlich $14 \mathrm{mg}$ (Min. $10 \mathrm{mg}$, Max. $17 \mathrm{mg}$ ) in $100 \mathrm{ccm}$ Blut und der prozentuale Anteil des Urea-N am Rest- $\mathrm{N}$ durchschnittlich $45 \%$ mit den Grenzwerten von $32 \%$ und $35 \%$. Der Gehalt des Gesamt- und des Urea-N im 24 stündigen Harn Gesunder betrug durchschnittlich $7.9 \mathrm{~g}(4.1 \mathrm{~g} \sim 11,2 \mathrm{~g})$ bzw. $6.2 \mathrm{~g}(2.5 \mathrm{~g} \sim 8.8 \mathrm{~g})$ und der prozentuale Anteil des Urea-N am Gesamt-N durchschnittlich 79\% (61 39\%).

Um die physiologische Schwankungen dieser N-Werte ein und derselben Person festzustellen, bestimmte ich bei allen die Werte im Blute 1 Woche später noch einmal und die im Harn täglich an 7 aufeinanderfolgenden Tagen. Der grösste Unterschied des Rest-N-Wertes im Blut ein und desselben Falles betrug $5 \mathrm{mg}$, der kleinste $1 \mathrm{mg}$, durchschnittlich also $3 \mathrm{mg}$ in $100 \mathrm{ccm}$ Blut. Der Unterschied des Urea-N im Blut betrug durchschnittlich $2 \mathrm{mg}$ in $100 \mathrm{ccm}$ Blut. Als absolute Werte des Rest-N fand ich bei Gesunden nie höhere Werte als $35 \mathrm{mg}$ in $100 \mathrm{ccm}$ Blut. Der durchschnittliche Unterschiedswert des Gesamt-N betrug $2 \mathrm{~g}$ und der grösste $3 \mathrm{~g}$.

\section{Ergebnisse bei Operationsfällen.}

Ich konnte die obigen Untersuchungen bei 45 Fällen von Magenresektion, 5 von Darmresektion oder Darmanastomose, 8 Gallenwegoperationen, 11 Appendektomie-Fällen und bei 10 sonstigen Operationen der Bauchhöhle, die meistenteils zu den sog. aseptischen Operationen gehörten, anstellen. Die Operationen wurden in Lumbal- sowie Lokalanästhsie durchgeführt. Es gab keinen Narkosenfall. 
Wie aus Tabelle I ersichtlich ist, waren die Werte des Rest- sowie des Urea-N im Blut bei meinen Untersuchungsfällen vor der Operation durchschnittlich ein wenig höher und die des Gesamt- und des Urea-N im 24 stündigen Harn dagegen etwas njedriger als die durchschnittlichen Werte bei Gesunden, abgesehen von den Appendektomie-Fällen, bei denen die Werte der betreffenden $\mathrm{N}$ meistens innerbalb der physiologischen Grenzen lagen. Zuweilen beobachtete ich merkliche Hyperazotämie, und zwar relativ häufig bei Magenkrebsfällen.

Nach der Operation steigen die Werte des Rest- und des Urea- $\mathrm{N}$ im Blut bei fast allen Fällen aller Operationsgruppen schon einen Tag postoperat. an und halten sich 3 Tage nach der Operation in fast gleicher Höhe, dann sinken sie verhältnismässig rasch ab, um 1 Woche postoperat. beinahe und 2 Wochen postoperat. völlig zum normalen Werte zurückzukehren. Die Werte des Rest- und des Urea-N bewegten sich dabei im grossen und ganzen parallel miteinander auf und $\mathrm{ab}$.

Der Gesamt-N-Gehalt des 24 stündigen Harns vermehrte sich bei den grössten Fällen auch schon einen Tag nach der Operation. Er nahm meistens weiter noch mehr zu oder hielt sich zuweilen in fast gleicher Höhe 3 Tage nah dem Eingriffe. Danach sank er verhältnismässig schnell ab, um schon 1 Woche postoperat. beinahe, und 2 Wochen postoperat. völlig zum Ausgangswerte abzufallen. Der Gehalt ein und desselben Harns an Urea-N schwankte dabei auch fast parallel mit dem an Gesamt-N.

- Eine Ausriahme bildete ein Fall von Magenresektion wegen Pylorusstenose infolge Magengeschwürs, bei welchem der Wert des Rest- und des Urea-N im Blut $36 \mathrm{mg}$ bzw. $18 \mathrm{mg}$ in $100 \mathrm{ccm}$ Blut vor der Operation betrug und das Maximum von $34 \mathrm{mg}$ bzw. $17 \mathrm{mg}$ einen Tage nach der Operation auftrat und sich darnach immer weiter verminderte, während sich der Harn-N nach der Operation vermehrte. Dieser Fall verlief postoperativ ausserordentlich günstig. Bei einem anderen Fall von Magenkrebs mit Pylorusstenosenerscheinungen und mit einer hochgradigen Eyperazotämie sank der Wert der beiden Blut-N einen Tage nach der Operation auf 61 bzw. $49 \mathrm{mg}$ vom Ausgangswert von $105 \mathrm{mg}$ bzw. $58 \mathrm{mg}$ in $100 \mathrm{ccm}$ Blut, stieg aber 3 Tage postoperat. auf $74 \mathrm{mg}$ bzw. $51 \mathrm{mg}$ in $100 \mathrm{ccm}$ Blut und verminderte sich darnach schnell, um 2 Wochen postoperat. zum Werte von $26 \mathrm{mg}$ bzw. $11 \mathrm{mg}$ in $100 \mathrm{ccm}$ Blut abzufallen. Bei diesem Fall schwankte der Harn-N nach der Operation wie bei den meisten Fällen.

Während ich eine postoperative Harn-N-Vermehrung bei fast allen Fällen keobachten konnte, sah ich doch 2 Ausnahmefälle. Bei einer 46 jahrigen Frau, bei der Cholecystektomie und Choledocholithotomie mit Hepaticusdrainage durchgeführt wurde, betrug z.B.' der Gesamt-N-Wert $6.4 \mathrm{~g}$ vor der Operation und ebenfalls $6.4 \mathrm{~g}$ einen Tag nach der Operation, 
TABLE I.

Werte der Blut-N und Harn-N vor und nach den Bauchoperationen.

\begin{tabular}{|c|c|c|c|c|c|c|c|c|}
\hline \multirow{2}{*}{ 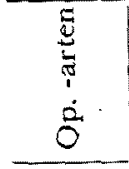 } & \multirow{2}{*}{ 泀语 } & & & \multirow{2}{*}{$\begin{array}{l}\text { vor d. Opera- } \\
\text { tion Durchs- } \\
\text { chnitt (Min.- } \\
\text { Max.) }\end{array}$} & \multicolumn{4}{|c|}{$\begin{array}{l}\text { Tage nach d. Operation } \\
\text { Durchschnitt (Min. -Max.) }\end{array}$} \\
\hline & & & & & 1 Tag & 3 Tage & $1 \mathrm{~W}$ & $2 \mathrm{~W}$ \\
\hline \multirow{4}{*}{ 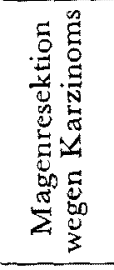 } & \multirow{4}{*}{28} & \multirow{2}{*}{$\begin{array}{c}\text { Blut } \\
(\mathrm{mg} / \mathrm{dl})\end{array}$} & Rest-N & $\begin{array}{c}34 \\
(19-105) \\
\end{array}$ & $\begin{array}{c}49 \\
(36-84) \\
\end{array}$ & $\begin{array}{c}49 \\
(33-114) \\
\end{array}$ & $\begin{array}{c}38 \\
(26-59)\end{array}$ & $\begin{array}{c}30 \\
(21-36)\end{array}$ \\
\hline & & & Uraa-N & $\begin{array}{c}17 \\
(8-58) \\
\end{array}$ & $\begin{array}{r}27 \\
(17-50) \\
\end{array}$ & $\begin{array}{c}27 \\
(14-53) \\
\end{array}$ & $\begin{array}{c}20 \\
(10-35) \\
\end{array}$ & $\begin{array}{c}13 \\
(8-24) \\
\end{array}$ \\
\hline & & \multirow{2}{*}{$\begin{array}{l}\text { Harn } \\
(\mathrm{g})\end{array}$} & Gesamt-N & $\begin{array}{c}6.4 \\
(2.3-12.7) \\
\end{array}$ & $\begin{array}{c}10.5 \\
(4.9-19.7) \\
\end{array}$ & $\begin{array}{c}12.6 \\
(3.7-19.1) \\
\end{array}$ & $\begin{array}{c}7.9 \\
(3.5-16.1)\end{array}$ & $\begin{array}{c}6,6 \\
(3.6-10.9)\end{array}$ \\
\hline & & & Urea-N & $\begin{array}{r}5.2 \\
(2.1-11.5) \\
\end{array}$ & $\begin{array}{r}8.6 \\
(3.7-16.0) \\
\end{array}$ & $\begin{array}{c}10.5 \\
(2.9-17.5) \\
\end{array}$ & $\begin{array}{c}6.4 \\
(2.1-13.8) \\
\end{array}$ & $\begin{array}{r}4.9 \\
(2.5-8.8) \\
\end{array}$ \\
\hline \multirow{4}{*}{ 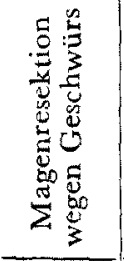 } & \multirow{4}{*}{17} & \multirow{2}{*}{$\underset{(\mathrm{mg} / \mathrm{dl})}{\text { Blut }}$} & Rest-N & $\begin{array}{c}34 \\
(25-44) \\
\end{array}$ & $\begin{array}{c}46 \\
(29-65) \\
\end{array}$ & $\begin{array}{c}40 \\
(26-54) \\
\end{array}$ & $\begin{array}{c}35 \\
(23-50) \\
\end{array}$ & $\begin{array}{r}30 \\
(24-44) \\
\end{array}$ \\
\hline & & & Urea-N & $\begin{array}{c}16 \\
(11-24)\end{array}$ & $\begin{array}{c}24 \\
(16-36) \\
\end{array}$ & $\begin{array}{c}20 \\
(13-32)\end{array}$ & $\begin{array}{c}16 \\
(11-26)\end{array}$ & $\begin{array}{c}13 \\
(9-21)\end{array}$ \\
\hline & & \multirow{2}{*}{$\begin{array}{l}\text { Harn } \\
(\mathrm{g})\end{array}$} & Gesamt-N & $\begin{array}{c}7.3 \\
(2.9-10.9) \\
\end{array}$ & $\begin{array}{c}12.7 \\
(4.5-20.9) \\
\end{array}$ & $\begin{array}{c}16.4 \\
(5.7-34.0) \\
\end{array}$ & $\begin{array}{r}9.5 \\
(4.0-13.7) \\
\end{array}$ & $\begin{array}{c}7.3 \\
(3.7-12.4) \\
\end{array}$ \\
\hline & & & Urea-N & $\begin{array}{r}6.2 \\
(2.4-8.7) \\
\end{array}$ & $\begin{array}{c}10.1 \\
(3.7-15.6)\end{array}$ & $\begin{array}{c}13.3 \\
(4.1-23.9)\end{array}$ & $\begin{array}{c}7.9 \\
(3.1-11.7)\end{array}$ & $\begin{array}{c}5.2 \\
(2.5-11.8)\end{array}$ \\
\hline \multirow{4}{*}{ 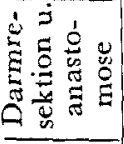 } & \multirow{4}{*}{5} & Blut & Rest-N & $3 \overline{6}$ & 53 & 43 & 37 & 31 \\
\hline & & $(\mathrm{mg} / \mathrm{dl})$ & Urea-N & 16 & 29 & 25 & 20 & 14 \\
\hline & & Harn & Gesamt-N & 5.1 & 10.7 & 10.4 & 6.7 & 5.8 \\
\hline & & $(\mathrm{g})$ & Urea-N & 4.1 & 8.4 & 8.7 & 5.2 & 4.6 \\
\hline \multirow{4}{*}{ 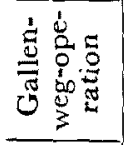 } & \multirow{4}{*}{8} & Blut & Rest-N & 32 & 45 & 48 & 38 & 28 \\
\hline & & $(\mathrm{mg} / \mathrm{dl})$ & Urea-N & $1 \overline{3}$ & 26 & 28 & 15 & 12 \\
\hline & & Harn & Gesamt-N & 6.8 & 7.2 & 9.0 & 6.9 & 5.8 \\
\hline & & (g) & Urea-N & 5.4 & 5.8 & 7.3 & 5.1 & 4.1 \\
\hline \multirow{4}{*}{ 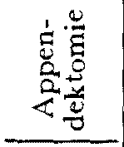 } & \multirow{4}{*}{11} & Blut & Kest-N & 31 & 52 & 46 & 34 & 30 \\
\hline & & $(\mathrm{mg} / \mathrm{dl})$ & Urea-N & 13 & 29 & 21 & 14 & 12 \\
\hline & & Harn & Gesamt-N & 9.0 & 12.7 & 13.6 & 8.6 & 7.9 \\
\hline & & (g) & Urea-N & 7.4 & 10.5 & 11.2 & 7.0 & 6.5 \\
\hline \multirow{4}{*}{ 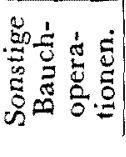 } & \multirow{4}{*}{10} & Blut & Rest-N & 39 & 54 & 51 & 35 & 28 \\
\hline & & $(\mathrm{mg} / \mathrm{dl})$ & Urea-N & 23 & 31 & 27 & 17 & 14 \\
\hline & & Harn & $\frac{\text { Gesamt-N }}{\text { Urea-N }}$ & 5.4 & 10.2 & 10.0 & 6.9 & $\frac{5.6}{4.4}$ \\
\hline & & & rea-N & 4.3 & 8.3 & 8.2 & 5.4 & 4.4 \\
\hline
\end{tabular}

und darnach verminderte er sich allmählich zum Wert von $4.5 \mathrm{~g} 2$ Wochen postoperat. Über diesen Fall soll später wieder berichtet werden. Bei einer anderen Frau von $26 \mathrm{Lj}$., bei der Notoperation wegen starker innerer Blutung infolge Ruptur der Tubenschwangerschaft durchgeführt wurde, zeigte der Harn- $\mathrm{N}$ während einer Woche nach der Operation dauernd Werte, die unter dem Ausgangswert lagen. Ausserdem fand ich bei 4 Fällen, nämlich bei 2 solchen von Magenkrebs, sowie je einem von Ulcus ventriculi und von Peritonitis tuberculosa eine Verminderung des Harn- $\mathrm{N}$ einen Tag der Operation, aber mehr oder minder auffallende Vermehrung desselben 3 Tage postoperat.

Nach Bürger u. Grauhan ${ }^{3 / 4)}$ u. a. ging das Ansteigen des Rest-N im Blut in der Regel Maximum der postoperativen Azoturie zeitlich voraus. 
Bei meinen 79 Fällen trat der Erhöhungsgipfel des Rest-N-Wertes sowie die grösste Ausscheidung des Gesamt-N im Harn einen Tag nach der Operation bei $20(25 \%)$ und der grösste Rest-N-Wert einen Tag, die grösste Gesamt-N-Ausscheidung 3 Tage nach der Operation bei 36 Fällen (46\%) auf. Im Ganzen stellte ich die maximale Erhöhung der N-Werte des Blutes bei 59 und die maximale Ausschiedung der N-Substanzen im Harn bei 25 Fällen einen Tag nach der Operation fest.

Bei den Untersuchungsfällen von $B$ ür ger und $G r$ a u han stieg der Rest-N-Wert des Blutes nach der Operation häufig auf das Doppelte, im Maximum auf das Vierfache des Ausgangswertes an. Die te ${ }^{5)}$ bestätigte auch das maximal 2- bis 3 fache Anwachsen der Ausgangswerte einiger N-Substanzen des Blutes nach der Operation. Bei meinen Fällen vermehrte sich in allen Gruppen der Rest-N des Blutes nicht so sehr wie bei den obengenannten Autoren und erreichte im Maximum durchschnittlich höchstens das 1.7 fache (bei Appendektomie) des Ausgangswertes, während einige Fälle nur ausnahmsweise im Maximum eine 2 bis 3 fache Erhöhung des Rest-N im Blut zeigten. Dagegen vermehrte sich der Gesamt-N im Harn bei meinen Fällen, abgesehen von der Gallenweggruppe, schon einen Tag nach der Operation auffallend stärker als bei den Fällen von Bürger u. Grauhan und erreichte im Maximum, meistens 3 Tage zuweilen aber schon einen Tag nach der Operation, durchschnittlich fast das Doppelte des Ausgangswertes. Besonders bemerkenswert war bei meinen Fällen, die in Lumbal- und Lokalanästhesie operiert wurden und bei denen sogar eine grössere Menge Flüssigkeit (täglich über 2 1) zugeführt wurde, dass die maximale N-Steigerung des Blutes nicht so hochgradig war, die N-Ausscheidung dagegen sich schon einen Tag postoperat. sehr auffallend vermehrte, während die Harnmenge sich mehr oder weniger verminderte. Bei den beiden genannten Autoren war die N-Ausscheidung einen Tag nach der Operation noch nicht stark, da die Diurese am Operationstage sehr oft sank trotz stärkerer Flüssigkeitszufuhr, was von ihnen zum Teil als Narkose-Effekt gedeutet wurde.

Der Einfluss der Narkose auf die postoperative Azotämie und Azoturie, vor allem auf die Azotämie wurde von Bürger u. Grauhan, Crainicianu u. a. ${ }^{6}$ und auch von Dietel völlig verneint oder doch wenigstens sehr gering eingeschätzt. Aber anderseits finden sich viele Arbeiten über den N-Stoffwechsel während und nach dèr Narkose. Eine Erhöhung der N-Ausscheidung nach Narkose, insbesondere nach Chloroformnarkose, wurde von mehreren Autoren (siehe Killia ${ }^{7 \prime)}$ festgestellt, während sie nach. Äthernarkose im allgemeinen als gering angesehen wurde. Ich will aber auf die Frage, ob die obigen Unterschiede zwischen meinen Ergebnissen und denen von Bürger u. Grauhan auf den Unterschied der Betäubungsart zurückzuführen seinen, nicht weiter eingehen, 
weil unter den von mir untersuchten Fällen kein Narkosefall ist.

Bei meinen Ergebnissen lässt sich die Verschiedenheit von denen der genannten Autoren vielleicht folgendermassen erklären. Die bei meinen Fällen nicht so hochgradige maximale Erhöhung des Rest-N im Blut kann möglicherweise zum Teil darauf zurückgeführt werden, dass bei ihnen die N-Ausscheidung im Harn schneller als bei den Fällen der geneanntn Auto$r \in n$ eintrat. Daraus geht als wahrscheinlich hervor, dass die parenterale Zufuhr grösserer Flüsigkeitsmengen bei der Operation, wie über 21 täglich bei meinen Fällen, nicht nur wesentlich als Nahrungs- oder als Mittel zur Steigerung der Herzfunktion, sondern auch zur Ausschwemmung der Eiweisszerfallprodukte diente, während die von mir gebrauchten spezifschen Diuretica keine auffallende Wirkung hatten.

Über die Frage, ob der postoperative Vermehrungsgrad des N-Gehaltes im Blut und Harn von der Grösse der Operationen abhängig ist, wird in der folgenden Mitteilung von $\mathrm{Zit} \mathrm{u} \mathrm{k} \mathrm{a} \mathrm{w} \mathrm{a} \mathrm{ausführlich} \mathrm{berichtet} \mathrm{werden.}$ Wenn ich trotzdem hier, wenn auch nur kurz über meine bei Bauchoperationen erhaltennen Resultate sprechen darf, so komme ich zum Schluss, dass Vermehrungsweise und -grad des Blut-, bzw. Harn-N nach der Operation von der Grösse, d.h. von der Ausdehnung und der Dauer der Operation nicht mitbestimmt ist. Wie aus der Tabelle I ersichtlich ist, ist sie, sowohl bei grösserer Operation wie Magenresektion, deren Operationsdauer meisten $80^{\prime}-100^{\prime}$ dauert, als auch bei kleineren wie Appendektomie, deren Dauer höchstens 20' bétrug, fast gleich. Nur bei der Operationsgruppe von Magenkrebs und von Gallensteinen bemerke ich im allgemeinen, dass das Ansteigen und Absteigen des Blut- $\mathrm{N}$ etwas protrahierte. Diese Protrahierung konnte dagegen bei der Magengeschwürgruppe, deren Operationsgrösse fast die gleiche wie bei Magenkrebs war, nicht festgestellt werden. Aus obigem Befund geht als wahrscheihlich hervor, dass das Protrahieren der postoperativen Azotämie bzw. Azoturie nicht durch die Operationsgrösse, sondern durch den schon vorher bestehenden Zustand der Opeierten verursacht wurde.

Bei der Gruppe von Gallenwegoperationen konstatierte ich, dass die postoperative Azoturie im allgemeinen nur geringgradig war. Oben berichtete ich als eine Ausnahme einen Fall von Gallenwegoperation, bei dem keine postoperative Azoturie auftrat. Bei Gallenwegfällen, besonders bei solchen mit Hepaticusdrainge, war die Harnmenge nach der Operation trotz der parenteralen Zufuhr grösserer Flüssigkeitmengen nicht vermehrt. Dies ist bei ihnen zum Teil darauf zurückzuführen, dass die Diurese wahrscheinlich infolge der schlechten Gallensekretion oder der Ableitung der Galle nach aussen, wie es schon von Clanssen ${ }^{8)}$ bestätigt wurde, vermindert war.

Weiter wiesen Bürger u. Grauhan darauf hin, dass Operationen 
TABLE II.

Postoperative maximale Steigerung des Rest-N-Wertes im Blut bei den Fällen mit und ohne Hyperazotämie.

\begin{tabular}{c|c|c|c}
\hline Gruppe & $\begin{array}{c}\text { Zahl d. } \\
\text { Fälle }\end{array}$ & $\begin{array}{c}\text { vor d. Operation } \\
\text { Durchschnitt } \\
\text { (Min. -Max.) }\end{array}$ & $\begin{array}{c}\text { nach der Operation } \\
\text { Durchschnitt } \\
\text { (Min. -Max.) }\end{array}$ \\
\hline A & 29 & $\begin{array}{c}42 \\
(35-105)\end{array}$ & $\begin{array}{c}58(38 \%) \\
(34-96)\end{array}$ \\
\hline B & 50 & $\begin{array}{c}30 \\
(19-35)\end{array}$ & $\begin{array}{c}50(67 \%) \\
(26-99)\end{array}$ \\
\hline
\end{tabular}

von Kranken mit primär erhöhtem Rest-N-Spigel, wie von Krebskranken, welche durchweg über die Norm erhöhte Ausgangswerte hatten, zu erheblichen Steigerungen der Rest-N-Quote des Blutes führten, was darnach auch von $\mathrm{D}$ i e t el bestätigt wurde. Von meinen Fällen waren die Rest-N-Werte bei $29 \mathrm{mehr}$ oder weniger höher als $35 \mathrm{mg}$ in $100 \mathrm{ccm}$ Blut (seihe Tabelle II A) und bei 50 Fällen normal (Tabelle II B). Der Rest-N-Wert des Blutes steigerte sich bei den ersteren nach der Operation im Maximum vom durchschnittlichen Ausgangswert $42 \mathrm{mg}$ in $100 \mathrm{ccm}$ Blut durchschnittlich auf $58 \mathrm{mg}$ in $100 \mathrm{ccm}$ Blut, so dass er eine durchschnittlich $36 \%$ ige Steigerung zeigte. Dagegen erhöhte er sich bei den letzteren nach der Operation im Maximum vom durchschnittlichen Ausgangswert $30 \mathrm{mg}$ durchschnittlich auf $50 \mathrm{mg}$, so dass er eine 67\% ige Steigerung zeigte. Das Maximum des postoperativ erhöhten Rest-N-Wertes war wie oben erwahnt bei einem Magenkrebs-Fall mit primär abnorm erhöhtem Wert sogar viel kleiner als der Ausgangswert. Also konnte ich eine erhebliche $\mathrm{N}$-Steigerung des Blutes von Kranken mit primär erhöhtem Rest-NWert nicht feststellen.

\section{Schluss.}

1. Bei fast allen 79 Bauchoperationen, bei welchen der Eingriff ohne postoperative Komplikationen verheilte, wurden postoperative Azotämie und Azoturie nachgewiesen. Sie traten meist schon einen Tag nach der Operation auf und hielten sich einige Tage lang. Vermehrungsweise und -grad der N-Substanz im Blut, bzw. im Harn waren von der Grösse, d.h. von der Ausdehnung und der Zeitdauer der Operation unabhängig. Nur bei Magenkrebss und Gallenstein-Fällen fand ich zuweilen ein mehr protrahiertes An- und Absteigen dieser N-Substanzen. Im Gegensatz B ü r g e r und Grauhan kommte ich bei Kranken mit primär erhöhtem Rest-NWert keine desiwegen besonders hochgradig aufsteigende Azotämie bestätigen.

2. Die quantitative Vermehrung dieser N-Substanz des Blutes war 
aber bei meiner Untersuchung nicht so stark wie bei den Fällen der oben erwähnten Autoren. Dies wurde zum Teil daraus erklärt, dass die vermehrte N-Ausscheidung meiner Fälle, bei denen grössere Mengen Flüssigkeit parenteral zugeführt wurden, sehr früh, nämlich schon einen Tag nach der Operation auftrat.

\section{Literatur.}

(1) Straaten, K., Arch. klin. Chir., 1940, 20,62-65.

(2) K a y a, H., Tohoku J. exp. Med., 1938, 33, 383-397.

(3) Bürger, M. u. M. Gra uhan, Ztschr. ges. exp. Med., 1923, 35, 16-42.

(4) Dieselbe, Ebendà, 1924, 42, 345-373.

(5) Dietel, F., Arch. klin. Chir., 1931, 163, 452-487.

(6) Crainicianu, A., M: Arnaud et I. Florian, J. de chir., 1927, 30, 394-405.

(7) Killian, H., Narkose zu operativen Zwecken, Berl., 1934, 117-126.

(8) Claussen, F., Ztschr. f. d. ges. exper. Med., 1923, 83, 231.

Diese Untersuchungen wurde unter finanzieller Unterstützung des Unterrichtsministeriums für wissenschaftliche Forschung ausgeführt, wofür ich meine herzlichsten Dank ausspreche. Prof. M. Muto. 